\title{
Evaluación ambiental de la producción a larga escala de micro- perlas de quitosano modificadas con tiourea como alternativa de valorización de residuos en el sector camaronicultor
}

\author{
Environmental assessment of large-scale production of \\ thiourea-modified chitosan microbeads as an alternative \\ for waste valorization in the shrimp farming sector
}

\author{
Samir I. Meramo-Hurtado ${ }^{1,+}$, Kariana A. Moreno Sader ${ }^{2,+}$ Ángel Darío González-Delgado ${ }^{3 *}$ \\ +Estos autores contribuyeron igualitariamente en este trabajo \\ ${ }^{1}$ Candidato a doctor, Doctorado en Ingeniería, Universidad de Cartagena, \\ Grupo de Investigación en Nanomateriales e Ingeniería de Procesos Asistida Por Computador (NIPAC), Cartagena de Indias, Colombia \\ ${ }^{2}$ Ingeniera Química, Programa de Ingeniería Química, Universidad de Cartagena, \\ Grupo de Investigación en Nanomateriales e Ingeniería de Procesos Asistida Por Computador (NIPAC), Cartagena de Indias, Colombia \\ ${ }^{3}$ Doctor en Ingeniera Química, Programa de Ingeniería Química, Universidad de Cartagena, \\ Grupo de Investigación en Nanomateriales e Ingeniería de Procesos Asistida Por Computador (NIPAC), Cartagena de Indias, Colombia \\ *agonzalezd1@unicartagena.edu.co
}

Cite this article as: J. D. Zorro Millán, I. A. Ruge Ruge, G. L. Camargo Millán "Bioreactor prototype: monitoring and control for the co-composting process from organic solid waste and sewage sludge", Prospectiva, Vol 18, N 1, 69-74, 2020.

Recibido: 17/09/2019 / Aceptado: 13/01/2020

https://doi.org/10.15665/rp.v18i1.2100

\begin{abstract}
RESUMEN
La generación de residuos durante el procesamiento del camarón es un alarmante problema de salud pública y medioambiental, lo que ha motivado a la búsqueda de alternativas de valorización de estos residuos tal como la producción de quitosano. Debido a sus múltiples propiedades, este biopolimero ha sido utilizado en diferentes sectores como el farmacéutico, tratamiento de aguas y biomedicina. En esta investigación, se realizó la simulación y evaluación ambiental de un proceso químico enfocado en la conversión de quitosano a partir del exoesqueleto de camarón hacia microperlas de quitosano modificadas con tiourea. Para ello, se utilizó el software de simulación Aspen Plus ${ }^{\circledR}$ y el algoritmo de reducción de residuos (WAR). Cuatro casos fueron considerados referentes a las contribuciones de energía y productos. El rendimiento de producción de las microperlas (MPs) fue de $2 \mathrm{~kg}$ MPs-Tiourea/kg quitosano. La evaluación ambiental mostró que el caso 3 alcanzó el valor más alto para impactos totales de salida $\left(1.23 \times 10^{+3} \mathrm{PEI} / \mathrm{h}\right)$, seguido por el caso 2, caso 1 y caso base. Se concluyó que la contribución de energía es menor a la del flujo de productos y que el proceso es amigable con el medio ambiente para las categorías de impactos atmosféricos $y$ toxicológicos.
\end{abstract}

Palabras claves: Evaluación ambiental, quitosano, microperlas, tiourea, simulación

\begin{abstract}
Waste generation during shrimp processing is a serious environmental and public health issue that has motivated the search for valorization alternatives such as chitosan extraction. Due to its properties, such biopolymer has been used in different sectors like pharmaceutic, water treatment and biomedicine. In this work, process simulation and environmental evaluation was carried out for the conversion of shrimp-derived chitosan into chitosan microbeads modified with thiourea. To this end, it was used the process simulation software Aspen Plus ${ }^{\circledR}$ and the waste reduction algorithm (WAR). Four cases were considered regarding contributions of energy and products. The conversion yield was calculated in $2 \mathrm{~kg} M P s-T h i o u r e a / \mathrm{kg}$ chitosan. The environmental evaluation showed that case 3 reached the highest total output impacts $\left(1.23 \times 10^{+3} \mathrm{PEI} / \mathrm{h}\right)$, followed by case 2, case 1 and base case. It was concluded that energy contributions are less than product contributions and the overall process is environmentally friendly for atmospheric and toxicological impacts.
\end{abstract}

Keywords: Environmental evaluation, chitosan, microbeads, thiourea, simulation. 


\section{INTRODUCCIÓN}

El desarrollo de los procesos industriales junto a la manufactura a larga escala en todo el mundo, ha generado un aumento en el consumo de recursos, los cuales generalmente son no renovables, lo anterior indica que se ha rebasado la capacidad que tiene la naturaliza para proveer estos recursos [1]. De otra forma, las actividades relacionadas a economías basadas en generación de bienes y servicios, han generado afectaciones sobre el ambiente y los ecosistemas [2]. En este sentido, se han afectado cuerpos de agua debido vertimientos con altas concentraciones de hidrocarburos y metales pesados, producto de las operaciones y procesos de sectores industriales tales como; manufactura, química/petroquímica, transporte, entre otros[3]. La industria camaronera, y por tanto la cadena acuícola, es uno de los sectores más afectados respecto al manejo los residuos y los potenciales impactos que esta genera sobre el ambiente. En el caso de Colombia, se estima una producción anual de 2400 toneladas de camarón, de los cuales el $20 \%$, corresponden los cuales son comúnmente descartados. A partir de estos residuos genera la producción de camarón, existe un potencial para la generación a escala industrial de quitosano, debido que para la composición del exoesqueleto de camarón (principal residuo de este producto), se reporta una concentración entre $30 \%$ y $40 \%$ p/p de quitina, siendo este último el principal precursor para la producción de quitosano, a través del proceso de desacetilación [4].

Las aplicaciones del quitosano como producto final son diversas, esta sustancia es utilizada en los procesos de la industria cosmetológica, alimenticia, entre otros, además, puede ser utilizada como materia prima para la producción de absorbentes (en forma de microperlas) para la remoción de contaminantes en sistemas de tratamiento de aguas residuales [5]. Las propiedades fisicoquímicas de las microperlas quitosano permiten que se comporte como un policatión, dándole alta capacidad de adsorción, por lo que el uso de materiales a base de esta sustancia se considera como una alternativa factible para la remoción de HAPs en cuerpos de agua. Adicionalmente, las microperlas de quitosano, dependiendo del proceso de producción, pueden ser modificadas agregando sustancias (a través de mezcla física) que puedan aumentar la capacidad de adsorción y remoción de los contaminantes [6]. En este sentido, la modificación de las microperlas de quitosano con tiourea resulta en una interesante alternativa para procesos de tratamiento de aguas residuales. En este sentido, la tiourea tiene afinidad con los hidrocarburos aromáticos, por lo que la modificación de los bio-adsorbentes con esta sustancia resulta en un material con propiedades mejoradas de adsorción, alta selectividad de contaminantes, e incluso mejora en la eficiencia en los procesos de recuperación del material [7].
Debido a que estas tecnologías se encuentran en fase de desarrollo, muchos de estos procesos solo están reportados a escala laboratorio. Por tanto, es de suma importancia evaluar la producción a escala industrial y aspectos relativos al desempeño ambiental de estos diseños, ya que un diseño de proceso u operación fisicoquímica que genere altos impactos ambientales, a pesar que pueda ser factible, no se considera sostenible [8]. En este sentido, el uso de herramientas computacionales para el escalamiento, simulación y evaluación de impactos ambientales de procesos se constituyen en alternativas para el desarrollo de procesos químicos que desde su etapa de diseño conceptual considera factores tales como: Eficiencia de producción, tasa de generación/consumo de impactos ambientales, contribución de las fuentes de energía a los impactos ambientales generados, entre otros, en miras de diagnosticar topologías más sostenibles e incluso, se puedan identificar oportunidades de mejora [9]. Se han reportado en la literatura estudios relacionados a la evaluación y simulación de procesos utilizando herramientas computacionales, por ejemplo; Moncada et al. [10] evaluaron dos topologías de biorrefinería para la producción de biodiesel a partir de microalgas y caña de azúcar, a través de análisis ambiental y técnico-económico. Cambero et al. [11] propusieron una optimización para cadena de suministro de biorrefinería incorporando evaluación económica y análisis ciclo de vida (ACV). Pernett-Bolaño et al. [12] simularon la oxidación catalítica del dióxido de azufre mediante la herramienta computacional de MATLAB y validaron los resultados con el software Aspen HYSYS.

Por otro lado, García et al. [13] evaluaron rutas para el aprovechamiento de residuos agroindustriales para producción de energía a través de análisis energético y ambiental. La importancia de las metodologías de evaluación de procesos a través de análisis ambiental va relacionada a que a partir de estas, se pueden evaluar diferentes alternativas para generación de un mismo producto considerando el desempeño ambientales de estas, en ese sentido; AlonsoFariñas et al. [14] utilizaron análisis ambiental para comparar procesos de producción de bio-etileno a través de rutas termoquímicas. Moreno-Sader et al. [15] evaluaron diferentes materias primas y rutas para producción de bio-oil, utilizando el algoritmo de reducción de residuo (WAR por sus siglas en ingles) junto al análisis exergético. Smith et al. [16] utilizaron indicadores de sostenibilidad (dentro de los cuales se considera el criterio ambiental) a través de la metodología GREENSCOPE desarrollada por la Agencia de Protección ambiental de los Estados Unidos (EPA por sus siglas en ingles) para evaluar y ajustar las condiciones de operación para un proceso de producción de benzaldehído y acido benzoico a partir de acido acético y tolueno. De acuerdo con lo anterior, existe una motivación por aplicar métricas de evaluación de procesos químicos utilizando 
criterio ambiental en términos de sostenibilidad, en miras de generar topologías para producción mas limpia, o diagnosticar oportunidades de mejora para estos, a través de herramientas computacionales. Por tanto, en este estudio se evaluó a través de análisis ambiental un proceso para producción de microperlas de quitosano modificadas con tiourea con el fin de diagnosticar oportunidades de mejora en miras del desarrollo a larga escala para este tipo de tecnologías basadas en el uso sostenible de residuos y aprovechamiento de recursos renovables.

\section{METODOLOGÍA}

\subsection{Descripción del proceso}

El proceso de producción de microperlas (MPs) de quitosano modificadas con tiourea consta de 4 etapas como se muestra en la Figura 1. Inicialmente se alimenta 77.61 $\mathrm{kg} / \mathrm{h}$ de quitosano y una disolución de ácido acético a un mezclador para la formación del gel de quitosano. Posteriormente, se realiza la modificación del gel a través de una mezcla física con tiourea. Esta nueva mezcla es enviada a un sistema de agitación (con ultrasonido) y se agrega una solución de $\mathrm{NaOH}$ (concentración $5 \mathrm{M}$ ) para elevar el pH. Lo anterior permite formar las perlas de quitosano modificadas con tiourea. Para eliminar dichas impurezas y estabilizar el $\mathrm{pH}$, se lavan las microperlas modificadas en tanques lavadores (con abundante agua). Finalmente, las perlas son enviadas a un secador con el fin de reducir la humedad de estas. El rendimiento de producción de las microperlas (MPs) fue de $2 \mathrm{~kg}$ MPs-Tiourea/kg quitosano.

Figura 1. Diagrama de bloques del proceso de producción de MPs de quitosano modificadas con tiourea

Figure 1. Block diagram of the production of chitosan microbeads modified with thiourea

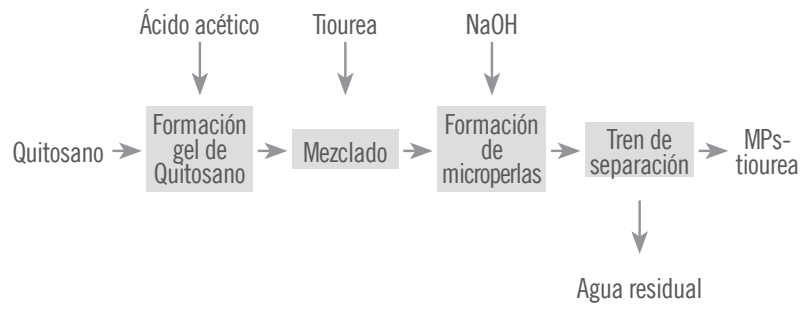

Para el proceso de producción de MPs de quitosano, se desarrolló el diagrama de bloques y la simulación del proceso a partir de las etapas y la información recolectada de la síntesis del producto a escala laboratorio. Experimentalmente se prepararon las microperlas de quitosano modificadas con tiourea mediante procesos de mezclado continuo y adición gota a gota de la mezcla en una solución de $\mathrm{NaOH}$ para la formación estructural como microperlas. Se realizó un inventario de materias primas usadas en los experimentos, con lo cual fue posible escalar el proceso. Para la simulación del proceso se utilizó la ingeniería de procesos asistida por computador a través del software Aspen Plus ${ }^{\circledR}$ conforme a lo reportado en publicaciones previas de los autores [6].

\subsection{Análisis ambiental basado en WAR}

Luego de la simulación del proceso, se desarrolló el análisis ambiental de la producción de microperlas de quitosano modificadas con tiourea. Para ello, fue necesaria la información reportada en la simulación de los balances extendidos de masa, energía y la información de los equipos y diseño de la planta. El análisis ambiental se realizó mediante el algoritmo de reducción de residuos (WAR, por sus siglas en inglés) con el software WAR GUI ${ }^{\circledR}$. Esta es una metodología comúnmente utilizada para desarrollar evaluaciones ambientales de procesos químicos. Esta herramienta asistida por computador fue desarrollada por la Agencia de Protección Ambiental, y permite cuantificar la tasa de generación (o consumo) de impactos ambientales potenciales (PEI, por sus siglas en ingles) para los procesos teniendo en cuenta los flujos de energía y productos [17]. La Tabla 1 muestra las expresiones matemáticas para estimar las categorías evaluadas por el algoritmo. Como se reporta en esta tabla, el algoritmo WAR evalúa el desempeño ambiental a través de 8 diferentes categorías de impacto, toxicológicas: toxicidad humana por ingestión (HTPI), toxicidad humana por exposición dérmica (HTPE), potencial de toxicidad acuática (ATP) y potencial de toxicidad terrestre TTP, atmosféricas: potencial de calentamiento global (GWP), Potencial de agotamiento del ozono (ODP), Potencial de oxidación fotoquímico (PCOP) y Potencial de acidificación (AP) [18].

Tabla 1. Expresiones matemáticas para la estimación de categorías en el algoritmo WAR. Fuente: [19] Table 1. Mathematical equations to estimate the categories of WAR algorithm. Source: [19]

\begin{tabular}{|c|c|c|c|}
\hline \multicolumn{4}{|c|}{ Categorías toxicológicas } \\
\hline Cat. & Ecuación & & Parámetro \\
\hline HTPI & $H T P I=\frac{1}{L D_{50}}$ & (1) & Dosis letal 50 oral aguda (LD50) \\
\hline HTPE & $H T P E=\frac{1}{T V L}$ & (2) & Valor límite de umbral (TLV) \\
\hline ATP & $H T P E=\frac{1}{L C_{50}}$ & (3) & Concentración letal (LC50) \\
\hline ПТ & $T T P=\frac{1}{L D_{50}}$ & (4) & Dosis letal 50 oral aguda (LD50) \\
\hline
\end{tabular}




\begin{tabular}{|c|c|c|}
\hline \multicolumn{3}{|c|}{ Categorías atmosféricas } \\
\hline Cat. & Ecuación & Parámetro \\
\hline GWP & $G W P=\frac{\int a_{i} c_{i}(t) d t}{\int a_{\mathrm{CO}_{2}} c_{\mathrm{CO}_{2}}(t) d t} m_{i}(5)$ & Emisión de dióxido de carbono $\left(\mathrm{a}_{\mathrm{CO}}\right)$ \\
\hline ODP & $O D P=\frac{d\left[O_{3}\right]_{i}}{d\left[O_{3}\right]_{i} F C K W-11} m_{i}$ & Concentración de ozono $\left(\mathrm{O}_{3}\right)$ \\
\hline PCOP & $P C O P=\frac{\frac{a_{i}}{b_{i}(t)}}{\frac{a_{C_{2} H_{4}}}{b_{C_{2} H_{4}}(t)}} m_{i}$ & Cambio en la emisión de etileno $\left(\mathrm{a}_{\mathrm{C}_{2}} \mathrm{H}_{4}\right)$ \\
\hline AP & $A P=\frac{\frac{V_{i}}{M_{i}}}{\frac{V_{\mathrm{SO}_{2}}}{M_{\mathrm{SO}_{2}}}} m_{i}$ & $\begin{array}{l}\text { Potencial de acidificación } \\
\text { para el } \mathrm{SO}_{2}\left(\mathrm{VsO}_{2}\right)\end{array}$ \\
\hline
\end{tabular}

\section{RESULTADOS Y DISCUSIÓN}

\subsection{Evaluación ambiental}

Para este proceso se desarrolló un análisis ambiental basado en la metodología WAR. Los balances de masa y energía obtenidos de la simulación permiten estimar los impactos ambientales potenciales para este proceso de producción de MPs de quitosano modificados con tiourea. Se consideraron cuatro casos de estudio para estudiar las contribuciones de los productos y la energía en la generación de impactos ambientales potenciales:

1. Caso base: sin considerar contribución de flujo de productos ni energía

2. Caso 1: considerando la contribución de energía y no de flujo de productos

3. Caso 2: considerando la contribución de flujo de productos y no de energía

4. Caso 3: considerando la contribución de energía y la contribución de flujo de productos

La Figura 2 muestra la salida y la tasa de generación de PEI para este caso de estudio. Se observa que este proceso consume impactos ambientales de acuerdo con la tasa de generación total de PEI para los casos base, 1 y $2\left(-5.84 \times 10^{+2}\right.$, $\left.-4.29 \times 10^{+2},-9.25 \times 10^{+1} \mathrm{PEI} / \mathrm{h}\right)$, mientras que para el caso 3 se obtuvo una generación moderadamente baja de impactos $\left(6.23 \times 10^{+1} \mathrm{PEI} / \mathrm{h}\right)$. Lo anterior indica que la operación tiene un desempeño ambiental amigable y es favorable en términos de sostenibilidad. En cuanto a los impactos totales de salida de PEI, se observa que el caso 3 alcanzó el valor más alto $\left(1.23 \times 10^{+3} \mathrm{PEI} / \mathrm{h}\right)$, seguido por el caso $2\left(1.08 \times 10^{+3}\right.$ $\mathrm{PEI} / \mathrm{h})$, caso $1\left(7.41 \times 10^{+2} \mathrm{PEI} / \mathrm{h}\right)$ y caso base $\left(5.86 \times 10^{+2}\right.$ $\mathrm{PEI} / \mathrm{h})$. De estos resultados, se concluye que la contribución de energía es menor a la contribución de flujo de produc- tos, por tanto, las microperlas de quitosano modificadas con tiourea aportan mayores impactos al medio ambiente debido a la naturaleza de las especies químicas que la componen.

Figura 2. Impactos ambientales globales para producción a larga escala de MPs de quitosano modificados con tiourea Figure 2. Global environmental impacts of large-scale production of chitosan microbeads modified with thiourea

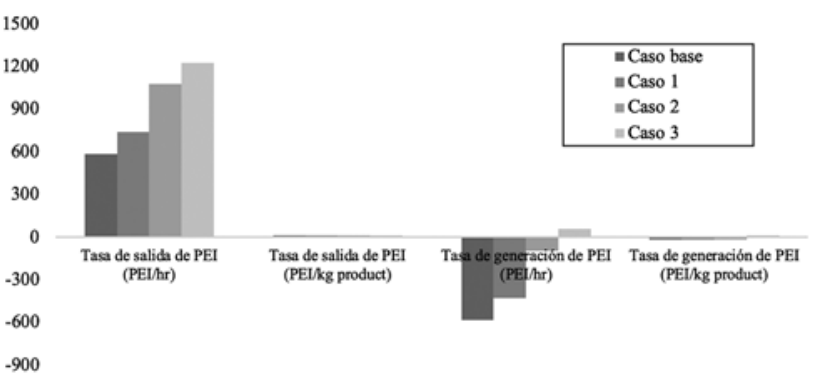

La Figura 3 muestra los impactos toxicológicos generados y la tasa de producción de PEI para la producción de las microperlas. Los impactos ambientales de salida afectan considerablemente a las categorías HTPI, HTPE y TTP para todos los escenarios en comparación con la categoría ATP. Los valores bajos en el potencial de toxicidad acuática para la salida de PEI (5.04 PEI/h para el caso base, 7.36 $\mathrm{PEI} / \mathrm{h}$ para el caso $1,2.30 \times 10^{+1} \mathrm{PEI} / \mathrm{h}$ para el caso $2 \mathrm{y}$ $2.53 \times 10^{+1}$ para caso 3 ) indican que este proceso de síntesis de microperlas a larga escala no representa riesgo alguno para la vida acuática y el producto obtenido no es tóxico para estas especies. Bertel y colaboradores [7] realizaron la evaluación ambiental del procesos de síntesis de microperlas de quitosano modificadas con magnetita y tiourea y reportaron resultados similares a los obtenidos en esta investigación. En su estudio, los autores obtuvieron valores más altos para las categorías de TTP y HTPI. Por otro lado, la generación de PEI para las categorías toxicológicas muestran un valor negativo sugiriendo que el proceso tiene, en su corriente de producto, menos químicos tóxicos que los alimentados al sistema, lo que confirma un desempeño ambiental favorable.

figura 3. Impactos ambientales de categorías toxicológicas para la producción a larga escala de MPs de quitosano modificadas con tiourea figure 3. Environmental impacts of toxicological categories for large-scale production of chitosan microbeads modified with thiourea

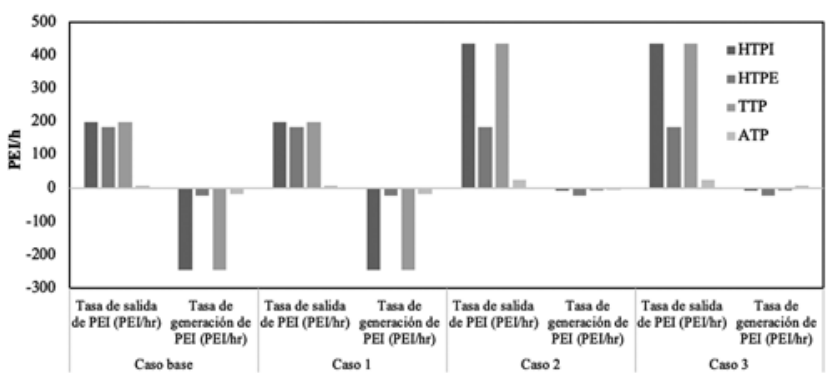


La Figura 4 muestra la velocidad de generación de impactos en las categorías de impacto atmosférico para la producción de las microperlas. Esta evaluación está compuesta por impactos globales: GWP y ODP, e impactos regionales: AP y PCOP. Los resultados obtenidos muestran que el proceso de producción de MPs de quitosano modificados con tiourea globalmente tiene un buen desempeño ambiental para las categorías atmosféricas. Específicamente, para el caso base y caso 2, la tasa de salida de PEI para GWP, ODP y AP fue igual a cero, lo que indica que los productos obtenidos en el proceso de síntesis no impactan al medio ambiente por calentamiento global, acidificación o agotamiento de ozono. Para los casos 1 y 3 se observan los máximos valores en la categoría de AP $\left(13.9 \times 10^{+1}\right.$ para ambos casos $)$ indicando que la fuente de energía utilizada en el proceso afecta a la atmósfera con el fenómeno de lluvia ácida atribuida a la presencia de azufre en el combustible. Además, es importante mencionar que este caso de estudio consume impactos ambientales relacionados con la categoría de PCOP, lo que implica que la transformación del quitosano y la tiourea en microperlas de quitosano modificadas mejora las propiedades químicas de las sustancias responsables del potencial de oxidación fotoquímico.

Figura 4. Impactos ambientales de categorías atmosféricas para la producción a larga escala de MPs de quitosano modificadas con tiourea Figure 4. Environmental impacts of atmospheric categories for large-scale production of chitosan microbeads modified with thiourea

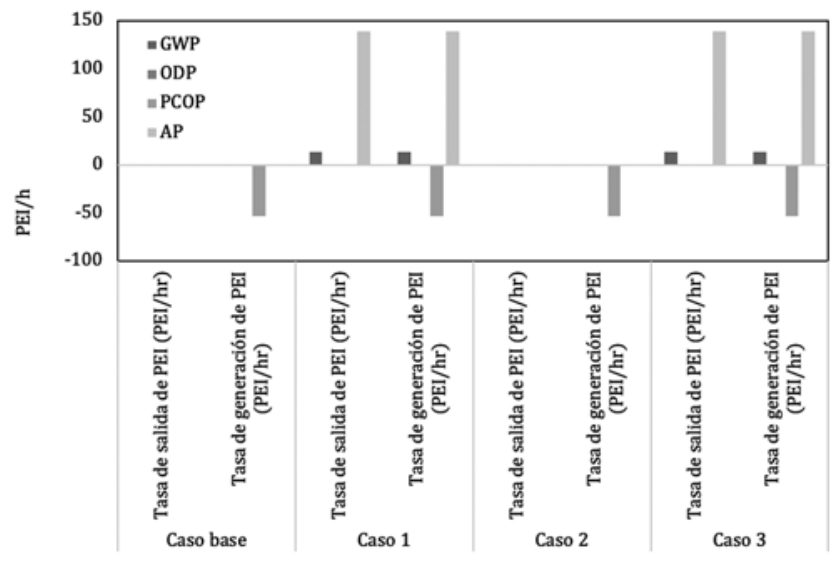

\section{CONCLUSIONES}

El desarrollo de esta investigación estuvo enfocado en la evaluación ambiental de la producción de microperlas de quitosano modificadas con tiourea. Se evaluaron cuatro casos para identificar la contribución de la energía y los productos en la generación y consumo de impactos ambientales. Se obtuvo que la contribución de la energía es menor a la del flujo de productos dado que los casos 3 y 2 alcanzaron los valores más altos para la salida de PEI $\left(1.23 \times 10^{+3} \mathrm{y}\right.$ $1.08 \times 10^{+3} \mathrm{PEI} / \mathrm{h}$, respectivamente). Además, se concluyó que el proceso es amigable ambientalmente debido a que la tasa de generación total de PEI es negativa para los casos base, 1 y $2\left(-5.84 \times 10^{+2},-4.29 \times 10^{+2},-9.25 \times 10^{+1} \mathrm{PEI} / \mathrm{h}\right)$. Se obtuvieron valores bajos en el potencial de toxicidad acuática para la salida de PEI en todos los casos indicando que el proceso no es nocivo para la vida acuática. En cuanto a las categorías de impactos atmosféricos, se observó que GWP, ODP y AP alcanzaron valores de cero para la tasa de salida de PEI en los casos base y caso 2 , mientras que para los casos 1 y 3 se obtuvieron los máximos valores de AP indicando que la fuente de energía afecta la atmosfera con el fenómeno de lluvia ácida.

\section{AGRADECIMIENTOS}

Los autores expresan su gratitud a la Universidad de Cartagena y al Departamento Administrativo de Ciencia, Tecnología e Innovación (COLCIENCIAS) por financiar la ejecución de esta investigación.

\section{CONFLICTOS DE INTERÉS}

Los autores no presentan ningún tipo de conflicto de interés que podrían influir de manera inapropiada en este trabajo.

\section{REFERENCIAS}

[1] V. Hernández, J. M. Romero-García, J. A. Dávila, E. Castro, and C. A. Cardona, "Techno-economic and environmental assessment of an olive stone based biorefinery," Resources, Conservation and Recycling, vol. 92, pp. 145-150, 2014.

[2] H. Rüstemo囚lu and A. R. Andrés, "Determinants of CO2emissions in Brazil and Russia between 1992 and 2011 : A decomposition analysis," Environmental Science and Policy, vol. 58, pp. 95-106, 2016.

[3] B. Bao, "Process Design and Optimization of Biorefining Pathways," Texas A\&M University, no. Ph.D. Dissertation, 2012.

[4] H. Bonfante-Alvarez, G. De Avila-Montiel, K. Cogollo-Herrera, M. Torrenegra-Alarcón, and A. Gonzalez-Delgado, "Evaluation of Five Chitosan Production Routes with Astaxanthin Recovery from Shrimp Exoskeletons," vol. 70, p. In press, 2018.

[5] S. Meramo-Hurtado, N. Urbina-Suarez, and Á. González-Delgado, "Computer-aided environmental and exergy analyses of a large-scale production of chitosan microbeads modified with $\mathrm{TiO} 2$ nanoparticles," Journal of Cleaner Production, vol. 273, 2019.

[6] S. Meramo-Hurtado, A. Herrera-Barros, and Á. Gonzalez-Delgado, "Evaluation of Large-Scale Production of Chitosan Microbeads Modified with Nanoparticles Based on Exergy Analysis," Energies, vol. 12, no. 1200, pp. 1-16, 2019.

[7] F. Bertel, G. Cogollo, S. Meramo-Hurtado, and A. González-Delgado, "Computer aided environmental analysis of a large-scale production of chitosan micro-beads modified with thiourea and magnetite," IOP Conference Series: Materials Science and Engineering, vol. 519, p. $012003,2019$.

[8] G. J. Ruiz-Mercado, R. L. Smith, and M. A. Gonzalez, "Sustainability indicators for chemical processes: I. Taxonomy," Industrial and Engineering Chemistry Research, vol. 51, no. 5, pp. 2309-2328, 2012. 
[9] R. Herrera-aristizábal, J. S. Salgado-dueñas, and Y. Yolima, "Environmental Evaluation of a Palm-based biorefinery under North-Colombian Conditions," Chemical Engineering Transactions, vol. 57, pp. 193-198, 2017.

[10] J. Moncada, J. A. Tamayo, and C. A. Cardona, "Integrating first, second, and third generation biorefineries: Incorporating microalgae into the sugarcane biorefinery," Chemical Engineering Science, vol. 118, pp. 126-140, 2014.

[11] C. Cambero, T. Sowlati, and M. Pavel, "Economic and life cycle environmental optimization of forest-based biorefinery supply chains for bioenergy and biofuel production," Chemical Engineering Research and Design, vol. 107, pp. 218-235, 2016.

[12] L. P. Bolaño, I. A. O. Martínez, M. E. R. Barrios, and C. A. R. Durán, "Modelamiento y simulación de un reactor catalítico para la oxidación de SO2 a SO3, utilizando V2O5 como catalizador Modeling and simulation of a catalytic reactor for the oxidation of $\mathrm{SO} 2$ to $\mathrm{SO} 3$, using V2O5 as catalyst," Prospectiva, vol. 14, no. 1, pp. 39-46, 2016.

[13] C. A. García, Á. Peña, R. Betancourt, and C. A. Cardona, "Energetic and environmental assessment of thermochemical and biochemical ways for producing energy from agricultural solid residues: Coffee Cut-Stems case," Journal of Environmental Management, 2017.

[14] B. Alonso-Fariñas, A. Gallego-Schmid, P. Haro, and A. Azapagic, "Environmental assessment of thermo-chemical processes for bio-ethylene production in comparison with bio-chemical and fossil-based ethylene," Journal of Cleaner Production, vol. 202, pp. 817-829, 2018.

[15] K. Moreno-Sader, S. I. Meramo-Hurtado, and A. D. González-Delgado, "Computer-aided environmental and exergy analysis as decisionmaking tools for selecting bio-oil feedstocks," Renewable and Sustainable Energy Reviews, vol. 112, no. February, pp. 42-57, 2019.

[16] R. L. Smith, G. J. Ruiz-Mercado, and M. A. Gonzalez, "Using GREENSCOPE indicators for sustainable computer-aided process evaluation and design," Computers and Chemical Engineering, vol. 81, pp. 272-277, 2015

[17] D. Young, R. Scharp, and H. Cabezas, "The waste reduction ( WAR ) algorithm : environmental impacts, energy consumption, and engineering economics," Waste Management, vol. 20, pp. 605-615, 2000.

[18] A. D. González-Delgado and Y. Y. Peralta-Ruíz, "Environmental Assessment of a Crude Palm Oil Production Process under North-Colombian conditions Using WAR Algorithm," International Journal of ChemTech Research, vol. 9 (12), pp. 833-843, 2016.

[19] D. Cassiani-Cassiani, D. A. Meza-González, and Á. D. González-Delgado, "Environmental Evaluation of Agar Production from Macroalgae Gracilaria sp.," Chemical Engineering Transactions, vol. 70, pp. 2005-2010, 2018 